\title{
On the canonical ideals of one-dimensional Cohen-Macaulay local rings *
}

\author{
Juan Elias ${ }^{\dagger}$
}

November 5, 2013

\begin{abstract}
In this paper we consider the problem of finding explicitly canonical ideals of one-dimensional Cohen-Macaulay local rings. We show that Gorenstein ideals contained in a high power of the maximal ideal are canonical ideals. In the codimension two case, from a Hilbert-Burch resolution, we show how to construct canonical ideals of curve singularities. Finally, we translate the problem of the analytic classification of curve singularities to the classification of local Artin Gorenstein rings with suitable length.
\end{abstract}

\section{Introduction}

Let $(R, \mathbf{m})$ be a one-dimensional Cohen-Macaulay local ring with maximal ideal $\mathbf{m}$ for which there exists a canonical module $\omega_{R}$, this is the case, for instance, if $R$ is the quotient of a local Gorenstein ring. Recall that $R$ possesses a canonical ideal (more precisely, the canonical module $\omega_{R}$ of $R$ exists and is contained in $R$ ) if and only if the total ring of fractions of the $\mathbf{m}$-adic completion of $R$ is Gorenstein, [13, Satz 6.21]. See [4, Chapter 3] for the basic properties of canonical modules and canonical ideals.

The question that motivates this paper is: can we describe explicitly canonical ideals? Recall that Boij in [3] addressed this problem for projective zero-dimensional schemes. A second question that we consider is: can we use canonical ideals for the analytic classification of singularities? In this paper we study these questions in the one dimensional case.

The contents of this paper is the following. In the second section we show that Gorenstein ideals contained in a high power of the maximal ideal are canonical ideals, Proposition 2.3. In the codimension two case, and following [3], from a Hilbert-Burch resolution we show how to construct canonical ideals for curve singularities, Proposition 2.8. In this section we recall how to describe "explicitly" the canonical module by using Rosenlicht's regular differential forms, [20, IV 9]. This strategy is very useful in the case of branches, and specially in the case case of monomial curve singularities. In the third section we address the problem of the analytic classification of curve singularities. We show canonical ideals $I \subset \mathcal{O}_{(X, 0)}$, where $X$ is a curve singularity, for which we compute the multiplicity and the socle degree of the Artin Gorenstein quotient $\mathcal{O}_{(X, 0)} / I$, Proposition 3.4. In Theorem 3.5 we translate the problem of the analytic classification of curve singularities $X$ to the classification of local Artin Gorenstein rings $\mathcal{O}_{(X, 0)} / I$ of suitable length.

Notations: Let $(R, \mathbf{m})$ be a one-dimensional Noetherian local ring with maximal ideal $\mathbf{m}$ and residue filed $\mathbf{k}=R / \mathbf{m}$. If $I$ is a $\mathbf{m}$-primary ideal of $R$ we denote by $\mathrm{HF}_{I}^{1}(n)=\operatorname{length}_{R}\left(R / I^{n+1}\right)$ the HilbertSamuel function of $I$. Hence there exist integers $e_{0}=(I) \geq 1$ and $e_{1}(I)$ such that $\operatorname{HP}_{I}^{1}(n):=e_{0}(I)(n+$ $1)-e_{1}(I)$, this is the Hilbert-Samuel polynomial of $I$, i.e. $\mathrm{HF}_{I}^{1}(n)=\mathrm{HP}_{I}^{1}(n)$ for $n \gg 0$. The integer $e_{0}(I)$

\footnotetext{
*2010 Mathematics Subject Classification. Primary 14.18; Secondary 13H15, 13H10;

Key words and Phrases: Canonical ideal, Gorenstein, Cohen-Macaulay, Hilbert-Samuel Function.

†Partially supported by MTM2010-20279-C02-01 and PRX12/00173
} 
is the multiplicity of $I$. We denote by $\mathrm{HF}_{I}^{0}(n)=$ length $h_{R}\left(I^{n} / I^{n+1}\right)$ the 0 -th Hilbert-Samuel function of $I$. Then $\mathrm{HP}_{I}^{0}(n)=e_{0}(I)$ for $n \gg 0$, the postulation number of $I$ is the least integer $\operatorname{pn}(I)$ such that $\mathrm{HF}_{I}^{0}(n)=\mathrm{HP}_{I}^{0}(n)$ for all $n \geq \operatorname{pn}(I)$. We set $\mathrm{HF}_{R}^{i}=\mathrm{HF}_{\mathbf{m}}^{i}, \mathrm{HP}_{R}^{i}=\mathrm{HP}_{\mathbf{m}}^{i}, i=0,1$, and $\operatorname{pn}(R)=\operatorname{pn}(\mathbf{m})$. If $M$ is an Artin $R$-module we define the socle degree $s(M)$ of $M$ as the last integer $t$ such that $\mathbf{m}^{t} M \neq 0$. The socle of $M$ is by definition the $R$-submodule $\operatorname{soc}(M)=\left(0:_{M} \mathbf{m}\right)$ of $M$.

Next we recall some basic facts of curve singularities. Let $(X, 0)$ be a reduced curve singularity of $\left(\mathbb{C}^{n}, 0\right)=\operatorname{Spec}\left(\mathcal{O}_{\left(\mathbb{C}^{n}, 0\right)}\right)$, i.e. $\mathcal{O}_{\left(\mathbb{C}^{n}, 0\right)}=\mathbb{C} \llbracket x_{1}, \ldots x_{n} \rrbracket$, and $(X, 0)=\operatorname{Spec}\left(\mathcal{O}_{(X, 0)}\right)$ where $\mathcal{O}_{(X, 0)}=$ $\mathcal{O}_{\left(\mathbb{C}^{n}, 0\right)} / I_{X}$ is a one-dimensional reduced ring with maximal ideal $\mathbf{m}_{X}$. We write $\mathrm{HF}_{X}^{i}=\mathrm{HF}_{\mathcal{O}_{(X, 0)}}^{i}$ and $\mathrm{HP}_{X}^{i}=\mathrm{HP}_{\mathcal{O}_{(X, 0)}}^{i}$. We assume that $n$ is the embedding dimension of $(X, 0)$, this is equivalent to say that $\mathbf{m}_{X} / \mathbf{m}_{X}^{2}$ is isomorphic as $\mathbb{C}$-vector space to the homogeneous linear forms of $P=\mathbb{C}\left[X_{1}, \cdots, X_{n}\right]$. Hence all element $x \in \mathbf{m}_{X} / \mathbf{m}_{X}^{2}$ defines an element in the quotient $\mathcal{O}_{(X, 0)}$ that we will denote again by $x$. Let $\nu: \bar{X}=\operatorname{Spec}\left(\overline{\mathcal{O}_{(X, 0)}}\right) \longrightarrow(X, 0)$ be the normalization of $(X, 0)$, where $\overline{\mathcal{O}_{(X, 0)}}$ is the integral closure of $\mathcal{O}_{(X, 0)}$ on its full ring $\operatorname{tot}\left(\mathcal{O}_{(X, 0)}\right)$ of fractions. The singularity order of $(X, 0)$ is $\delta(X)=\operatorname{dim}_{\mathbb{C}}\left(\mathcal{O}_{\bar{X}} / \mathcal{O}_{(X, 0)}\right)$. We denote by $\mathcal{C}$ the conductor of the finite extension $\nu^{*}: \mathcal{O}_{(X, 0)} \hookrightarrow \overline{\mathcal{O}_{(X, 0)}}$ and by $c(X)$ the dimension of $\overline{\mathcal{O}_{(X, 0)}} / \mathcal{C}$. Let $\omega_{(X, 0)}=\operatorname{Ext}_{\mathcal{O}_{\left(\mathbb{C}^{n}, 0\right)}}^{n-1}\left(\mathcal{O}_{(X, 0)}, \Omega_{\left.\mathcal{O}_{(\mathbb{C}}^{n}, 0\right)}^{n}\right)$ be the dualizing module of $(X, 0)$. We can consider the composition morphism of $\mathcal{O}_{(X, 0)}$-modules

$$
\gamma_{X}: \Omega_{(X, 0)} \longrightarrow \nu_{*} \Omega_{\bar{X}} \cong \nu_{*} \omega_{\bar{X}} \longrightarrow \omega_{(X, 0)} .
$$

Let $d: \mathcal{O}_{(X, 0)} \longrightarrow \Omega_{(X, 0)}$ the universal derivation, then we have a $\mathbb{C}$-map $\gamma_{X} d$ that we also denote by $d: \mathcal{O}_{(X, 0)} \longrightarrow \omega_{(X, 0)}$. The Milnor number of $(X, 0)$ is $\mu(X)=\operatorname{dim}_{\mathbb{C}}\left(\omega_{(X, 0)} / d \mathcal{O}_{(X, 0)}\right)$, [6]. Notice that $(X, 0)$ is non-singular iff $\mu(X)=0$ iff $\delta(X)=0$ iff $c(X)=0$.

In the following result we collect some basic results on $\mu$ and other numerical invariants that we will use later on.

Proposition 1.1. Let $(X, 0)$ be a reduced curve singularity of embedding dimension $n$. Then

(i) $\mu(X)=2 \delta(X)-r+1$, where $r$ is the number of branches of $(X, 0)$.

(ii) It holds

$$
e_{0}(X)-1 \leq e_{1}(X) \leq \delta(X) \leq \mu(X)
$$

and $e_{1}(X) \leq\left(\begin{array}{c}e_{0}(X) \\ 2\end{array}\right)-\left(\begin{array}{c}n-1 \\ 2\end{array}\right)$.

(iii) If $X$ is singular then $\delta(X)+1 \leq c(X) \leq 2 \delta(X)$, and $c(X)=2 \delta(X)$ if and only if $\mathcal{O}_{(X, 0)}$ is a Gorenstein ring.

Proof. (i) [6, Proposition 1.2.1]. (ii) [6, Proposition 1.2.4 (i)], [17], [9], [10]. (iii) [20, Proposition 7, pag. 80], and [2].

ACKNOWLEDGMENTS. The author thanks the referee for improving some results of section two.

\section{Canonical ideals}

The first aim of this section is to find conditions on a $\mathbf{m}$-primary ideal $I$ to be a canonical ideal.

Lemma 2.1. Let $(R, \mathbf{m})$ be a one-dimensional Cohen-Macaulay local ring and let $I$ be an $\mathbf{m}$-primary ideal of $R$. Let $x \in \mathbf{m}$ be a parameter of $R$.

(i) If $R / x^{n} I$ is a Gorenstein ring for some $n \geq 1$, then $R / I$ is a Gorenstein ring and $\left(x^{n} I:_{R} \mathbf{m}\right)=$ $x^{n}\left(I:_{R} \mathbf{m}\right)$.

(ii) Assume that $I \subset x R$. If $R / I$ is a Gorenstein ring, then $R / x^{n} I$ is a Gorenstein ring for all $n \geq 1$. 
Proof. (1) The short exact sequence

$$
0 \longrightarrow R / I \stackrel{x^{n}}{\longrightarrow} R / x^{n} I \longrightarrow R / x^{n} R \longrightarrow 0
$$

yields the exact sequence

$$
0 \longrightarrow \operatorname{Hom}_{R}(R / \mathbf{m}, R / I) \stackrel{x^{n}}{\longrightarrow} \operatorname{Hom}_{R}\left(R / \mathbf{m}, R / x^{n} I\right) \longrightarrow \operatorname{Hom}_{R}\left(R / \mathbf{m}, R / x^{n} R\right)
$$

of socles, which shows

$$
\operatorname{Hom}_{R}(R / \mathbf{m}, R / I) \stackrel{x^{n}}{\cong} \operatorname{Hom}_{R}\left(R / \mathbf{m}, R / x^{n} I\right),
$$

because $\operatorname{Hom}_{R}(R / \mathbf{m}, R / I) \neq 0$ and $\operatorname{Length}_{R}\left(\operatorname{Hom}_{R}\left(R / \mathbf{m}, R / x^{n} I\right)\right)=1$. Hence $R / I$ is a Gorenstein ring.

(ii) Let $\alpha \in\left(x^{n} I:_{R} \mathbf{m}\right)$. Then since $x \alpha \in x^{n} I \subset x^{n+1} R$, we get $\alpha \in x^{n} R$, which shows $\pi\left(\left(x^{n} I:_{R}\right.\right.$ m) $\left./ x^{n} I\right)=0$ where $\pi: R / x^{n} I \longrightarrow R / x^{n} R$ denotes the canonical epimorphism. Hence we get the isomorphism

$$
\operatorname{Hom}_{R}(R / \mathbf{m}, R / I) \stackrel{x^{n}}{\cong} \operatorname{Hom}_{R}\left(R / \mathbf{m}, R / x^{n} I\right),
$$

in the exact sequence

$$
0 \longrightarrow \operatorname{Hom}_{R}(R / \mathbf{m}, R / I) \stackrel{x^{n}}{\longrightarrow} \operatorname{Hom}_{R}\left(R / \mathbf{m}, R / x^{n} I\right) \longrightarrow \operatorname{Hom}_{R}\left(R / \mathbf{m}, R / x^{n} R\right)
$$

of socles. Thus Length $R\left(\operatorname{Hom}_{R}\left(R / \mathbf{m}, R / x^{n} I\right)\right)=1$, so that $R / x^{n} I$ is a Gorenstein ring for all $n \geq 1$.

Proposition 2.2. Let $I$ be an $\mathbf{m}$-primary ideal of $R$. Then $I$ is a canonical ideal of $R$ if and only if there exists a parameter $x \in \mathbf{m}$ of $R$ such that $R / x I$ is a Gorenstein ring.

Proof. We have only to prove the if part. Let $Q$ the total ring of fractions of $R$. Since $\mathrm{H}_{\mathbf{m}}^{1}(I) \cong Q / I$, it suffices to see $\operatorname{Length}_{R}\left(I:_{Q} \mathbf{m}\right) / I=1$. Let $\alpha \in\left(I:_{Q} \mathbf{m}\right)$. Then since $x \alpha \in I \subset R$ and $(x \alpha) \mathbf{m} \subset x I$, we have

$$
x \alpha \in\left(x I:_{R} \mathbf{m}\right)=x\left(I:_{R} \mathbf{m}\right)
$$

(see the proof of Lemma $2.1(\mathrm{i}))$. Hence $I \subsetneq\left(I:_{Q} \mathbf{m}\right) \subset\left(I:_{R} \mathbf{m}\right)$, so that

$$
\operatorname{Length}_{R}\left(I:_{Q} \mathbf{m}\right) / I=\operatorname{Length}_{R}\left(I:_{R} \mathbf{m}\right) / I=1
$$

as wanted.

In the next result we prove that an $\mathbf{m}$-primary Gorenstein ideal contained in a high power of the maximal ideal is a canonical ideal. Notice that this result cannot be extended to any $\mathbf{m}$-primary Gorenstein ideal. Let $R$ be a one-dimensional Cohen-Macaulay local ring of Cohen-Macaulay type 2 and embedding dimension $b \geq 3$, for instance $R=\mathbf{k} \llbracket t^{5}, t^{6}, t^{7} \rrbracket$. Then the maximal ideal is a Gorenstein ideal minimally generated by $b \geq 3$ elements. Since the minimal number of generators of a canonical ideal is the Cohen-Macaulay type of $R, \mathbf{m}$ is not a canonical ideal.

Corollary 2.3. Let $x \in \mathbf{m}$ and assume that $\mathbf{m}^{r+1}=x \mathbf{m}^{r}$ for some $r \geq 0$. Let $I$ be an $\mathbf{m}$-primary ideal of $R$ such that $I \subset \mathbf{m}^{r+1}$. If $R / I$ is a Gorenstein ring, then $I$ is a canonical ideal of $R$, whence $\operatorname{tot}(\widehat{R})$ is a Gorenstein ring.

Proof. The assertion follows from Lemma 2.1 and Proposition 2.2, since $I \subset \mathbf{m}^{r+1} \subset x R$.

Remark 2.4. Recall that $\operatorname{pn}(R) \leq e_{0}(R)-1$, [16, Proposition 12.14]. Hence, if $I \subset \mathbf{m}^{e_{0}(R)}$ is an $\mathbf{m}$-primary ideal such that $R / I$ is a Gorenstein ring then $I$ is a canonical ideal. 
Last result pointed out that a basic problem in Commutative Algebra is to find methods to construct Gorenstein ideals. We know that complete intersection ideals are Gorenstein; by a result of Serre, in codimension two to be Gorenstein is equivalent to be a complete intersection; in codimension three, Gorenstein ideals are the ideals generated by the Pfaffians of skew-symmetric matrices, [5]. In Proposition 2.8 we show how to construct canonical ideals, that are Gorenstein, from a Hilbert-Burch resolution. On the other hand, notice that if $I$ is a canonical ideal and $y \in \mathbf{m}$ is a non-zero divisor of $R$ then $y^{t} I, t \geq 1$, is a canonical ideal as well, but the length of $R / y I$ is not under control. In fact, for all $t \geq 1$ we have, [16, Theorem 12.5],

$$
\begin{aligned}
\operatorname{Length}_{R}\left(R / y^{t} I\right) & =\operatorname{Length}_{R}(R / I)+\operatorname{Length}_{R}\left(I / y^{t} I\right) \\
& =\operatorname{Length}_{R}(R / I)+t \operatorname{Length}_{R}(R /(y)) \\
& \geq \operatorname{Length}_{R}(R / I)+t e_{0}(R) .
\end{aligned}
$$

In the next result we will find canonical ideals for which we compute the multiplicity or the socle degree; in the second part we take $t \geq 4 \mu(X)+1$, where $(X, 0)$ is a reduced curve singularity, because we have to consider a high $t$ in Theorem 3.5. See Example 2.7 for an explicit application of the next result.

Proposition 2.5. Let $(X, 0)$ be a reduced curve singularity.

(i) Let $z$ be a degree $t \geq 2 \mu(X)+1$ superficial element of $\mathcal{O}_{(X, 0)}$. Then the $\mathcal{O}_{(X, 0)}$-module $z \omega_{(X, 0)}$ is a canonical ideal of $(X, 0)$ such that $\mathcal{O}_{(X, 0)} / z \omega_{(X, 0)}$ is a Gorenstein ring of colength te $e_{0}(X)-2 \delta(X)$.

(ii) For $t \geq 4 \mu(X)+1$ the socle degree of $\mathcal{O}_{(X, 0)} / z \omega_{(X, 0)}$ is at most

$$
e_{0}(X)(t-2 \mu(X)-1)+2 \delta(X)+e_{1}(X)+2(1-r) .
$$

This number is bounded above by $\delta(X)\left(4 e_{0}(X)+3\right)$.

(iii) If $(X, 0)$ is Gorenstein then $\mathcal{O}_{(X, 0)}$ is a canonical ideal and for every superficial element $z$ of degree $t \geq 1, z \mathcal{O}_{(X, 0)}$ is a canonical ideal.

Proof. (i) Since $\mathcal{O}_{(X, 0)}$ is a one-dimensional reduced ring we know that $\omega_{(X, 0)}$ is a sub- $\mathcal{O}_{(X, 0)}$-module of $\operatorname{tot}\left(\mathcal{O}_{(X, 0)}\right),[4,3.3 .18]$. Let us consider the perfect pairing, [20, Chapter IV],

$$
\begin{array}{ccccc}
\frac{\nu_{*} \mathcal{O}_{\bar{X}}}{\mathcal{O}_{(X, 0)}} & \times & \frac{\omega_{(X, 0)}}{\nu_{*} \Omega_{\bar{X}}} & \stackrel{\eta}{\longrightarrow} & \mathbb{C} \\
F & \times & \alpha & \longrightarrow & \sum_{i=1}^{r} \operatorname{res}_{p_{i}}(F \alpha)
\end{array}
$$

notice that for all $\lambda \in R$ it holds

$$
\eta(\lambda F, \alpha)=\sum_{i=1}^{r} \operatorname{res}_{p_{i}}(\lambda F \alpha)=\eta(F, \lambda \alpha) .
$$

Hence, since $\mathbf{m}^{c(X)}$ annihilates the quotient $\nu_{*} \mathcal{O}_{\bar{X}} / \mathcal{O}_{(X, 0)}$ we get that $\mathbf{m}^{c(X)}$ annihilates also the quotient $\omega_{(X, 0)} / \nu_{*} \Omega_{\bar{X}}$. Hence $\mathbf{m}^{c(X)} \omega_{(X, 0)} \subset \nu_{*} \Omega_{\bar{X}}=\nu_{*} \mathcal{O}_{\bar{X}}$. Again, since $\mathbf{m}^{c(X)} \nu_{*} \mathcal{O}_{\bar{X}} \subset \mathcal{O}_{(X, 0)}$ we get $\mathbf{m}^{2 c(X)} \omega_{(X, 0)} \subset \mathcal{O}_{(X, 0)}$. On the other hand the epimorphism of $\mathcal{O}_{(X, 0)}$-modules

$$
\frac{\omega_{(X, 0)}}{\mathcal{O}_{(X, 0)} d \mathcal{O}_{(X, 0)}} \longrightarrow \frac{\omega_{(X, 0)}}{\nu_{*} \Omega_{\bar{X}}}
$$

assures that $\mathbf{m}^{\mu(X)} \omega_{(X, 0)} \subset \nu_{*} \Omega_{\bar{X}}=\nu_{*} \mathcal{O}_{\bar{X}}$. Hence $\mathbf{m}^{c(X)} \nu_{*} \mathcal{O}_{\bar{X}} \subset \mathcal{O}_{(X, 0)}$

$$
\mathbf{m}^{\mu(X)+c(X)} \omega_{(X, 0)} \subset \mathcal{O}_{(X, 0)} .
$$

Let us consider the sequence

$$
\mathbf{m}^{t} \omega_{(X, 0)} \subset \mathcal{O}_{(X, 0)} \subset \nu_{*} \mathcal{O}_{\bar{X}}=\nu_{*} \Omega_{\bar{X}} \subset \omega_{(X, 0)} .
$$


From the perfect pairing of the beginning of the proof we get $\operatorname{dim}_{\mathbb{C}}\left(\omega_{(X, 0)} / \mathcal{O}_{(X, 0)}\right)=2 \delta(X)$. Since $z$ is a degree $t \geq 2 \mu(X)$ superficial element of $\mathcal{O}_{(X, 0)}$

$$
\operatorname{dim}_{\mathbb{C}}\left(\omega_{(X, 0)} / z \omega_{(X, 0)}\right)=t e_{0}(X)
$$

[16, Theorem 12.5]. From this identity and Proposition 1.1 we get the first part of the claim.

(ii) We have the following inequalities for $t \geq 4 \mu(X)+1$

$$
\begin{aligned}
s\left(\frac{\mathcal{O}_{(X, 0)}}{z \omega_{(X, 0)}}\right) & =s\left(\frac{\mathbf{m}^{2 \mu(X)+1}}{z \omega_{(X, 0)}}\right)+2 \mu(X)+1 \\
& \leq \operatorname{Length}\left(\frac{\mathbf{m}^{2 \mu(X)+1}}{z \omega_{(X, 0)}}\right)+2 \mu(X) \\
& =\operatorname{Length}\left(\frac{\mathcal{O}_{(X, 0)}}{z \omega_{(X, 0)}}\right)-\operatorname{Length}\left(\frac{\mathcal{O}_{(X, 0)}}{\mathbf{m}^{2 \mu(X)+1}}\right)+2 \mu(X) .
\end{aligned}
$$

From Proposition 1.1 and the first part of this result we get

$$
\begin{aligned}
s\left(\frac{\mathcal{O}_{(X, 0)}}{z \omega_{(X, 0)}}\right) & \leq\left(t e_{0}(X)-2 \delta(X)\right)-\left(e_{0}(2 \mu(X)+1)-e_{1}(X)\right)+2 \mu(X) \\
& =e_{0}(X)(t-2 \mu(X)-1)+2 \delta(X)+e_{1}(X)+2(1-r) .
\end{aligned}
$$

From Proposition 1.1 we get that the socle degree is bounded above by $\delta(X)\left(4 e_{0}(X)+3\right)$.

(iii) Since any superficial element is a non-zero divisor we get the claim.

Recall that it is possible to give an "explicit" description of $\omega_{(X, 0)}$ by using Rosenlicht's regular differential forms, [20, IV 9], [6, Section 1]. This strategy is very useful in the case of branches, specially in the case of monomial curve singularities.

We denote by $\Omega_{\bar{X}}\left(p\right.$.) the set of meromorphic forms in $\bar{X}$ with a pole at most in the set $\left\{p_{1}, \cdots, p_{r}\right\}$. Then Rosenlicht's differential forms are defined as follows: $\omega_{(X, 0)}^{R}$ is the set of $\nu_{*}(\alpha), \alpha \in \Omega_{\bar{X}}(p$.), such that for all $F \in \mathcal{O}_{(X, 0)}$

$$
\sum_{i=1}^{r} \operatorname{res}_{p_{i}}(F \alpha)=0
$$

Notice that we have a mapping that we also denote by $d_{R}: \mathcal{O}_{(X, 0)} \longrightarrow \omega_{(X, 0)} \longrightarrow \nu_{*} \Omega_{\bar{X}} \hookrightarrow \omega_{(X, 0)}^{R}$. In $[1$, Chap. VIII $]$ it is proved that $\omega_{(X, 0)} \stackrel{\phi}{\cong} \omega_{(X, 0)}^{R}$ and $d_{R}=\phi d$, where $d: \mathcal{O}_{(X, 0)} \longrightarrow \omega_{(X, 0)}$, see Section 1. From now on we assume that $(X, 0)$ is a branch, i.e. $r=1$. Let $t \in \operatorname{tot}\left(\mathcal{O}_{(X, 0)}\right)$ be a uniformizing parameter of $(X, 0)$, this means $\overline{\mathcal{O}_{(X, 0)}} \cong \mathbb{C} \llbracket t \rrbracket$. We can consider $\mathcal{O}_{(X, 0)}$ as a sub-C-algebra of $\mathbb{C} \llbracket t \rrbracket$ and then we may assume hat there exists a parametrization of $(X, 0)$

$$
\left\{\begin{array}{l}
x_{1}=t^{n_{1}} \\
x_{i}=f_{i}(t) \quad i=2, \cdots, n
\end{array}\right.
$$

with $n_{1}=e_{0}(X)$ and $\operatorname{val}_{t}\left(f_{i}\right) \geq n_{1}, i=2, \cdots, n$. Here $\operatorname{val}_{t}$ denotes the valuation with respect $t$ that is defined in $\operatorname{tot}\left(\mathcal{O}_{(X, 0)}\right)$. Notice that the conductor $\mathcal{C}$ of the extension $\mathcal{O}_{(X, 0)} \subset \overline{\mathcal{O}_{(X, 0)}}$ is in particular an ideal of $\overline{\mathcal{O}_{(X, 0)}} \cong \mathbb{C} \llbracket t \rrbracket$ so it is generated by $t^{c}$.

For all subset $N$ of $\operatorname{tot}\left(\mathcal{O}_{(X, 0)}\right)$ we denote by $\Gamma_{N}$ the the set of rational numbers $\operatorname{val}_{t}(a)$ for all $a \in N \backslash\{0\}$, we assume that $\Gamma_{N}$ contains the zero element.

The following result is well known. We include it here for the readers' convenience, see [11, Example 2.1.9]. 
Proposition 2.6. Let $(X, 0)$ be a branch. It holds $\Gamma_{\omega_{(X, 0)}} \subset \mathbb{Z} \backslash\left(-\Gamma_{X}-1\right)$, in particular $\Gamma_{\omega_{(X, 0)}} \subset-c+\mathbb{N}$ and there exists $\alpha \in \omega_{(X, 0)}$ such that $\operatorname{val}_{t}(\alpha)=-c$. If $(X, 0)$ is a monomial curve singularity then $\Gamma_{\omega_{(X, 0)}}=\mathbb{Z} \backslash\left(-\Gamma_{X}-1\right)$.

Proof. All $\alpha \in \omega_{(X, 0)}$ can be written $\alpha=t^{n} g(t) d t$ with $n \in \mathbb{Z}$ and $g(t) \in \mathbb{C} \llbracket t \rrbracket$ an invertible series. Then for all $F=t^{m} g(t) \in \mathcal{O}_{(X, 0)}, g(t) \in \mathbb{C} \llbracket t \rrbracket$ an invertible series, we get that res $(\alpha F)=0$. This implies in particular that $n+m \neq-1$, so $n \in \mathbb{Z} \backslash\left(-\Gamma_{X}-1\right)$. Let us consider an element $\alpha=t^{n} g(t) d t \in \omega_{(X, 0)}$, $g(t) \in \mathbb{C} \llbracket t \rrbracket$ an invertible series, with $n \leq-c-1$. Since $-n-1 \geq c$ we get $t^{-n-1} \in \mathcal{O}_{(X, 0)}$ and then a contradiction: $\operatorname{val}_{t}\left(\alpha t^{-n-1}\right)=-1$. The differential $\alpha=t^{-c} d t$ belongs to $\omega_{(X, 0)}$ and $\operatorname{val}_{t}(\alpha)=-c$.

Let assume now that $(X, 0)$ is a monomial curve singularity and let $n$ be an integer of $\mathbb{Z} \backslash\left(-\Gamma_{X}-1\right)$. Consider the differential $\alpha=t^{n} d t$, we only have to prove that $\alpha \in \omega_{(X, 0)}$. Let us consider $F=\sum_{i \geq 0} a_{i} t^{i}$ a power series with coefficients $a_{i} \mathbb{C}, i \geq 0$. Since $(X, 0)$ is monomial, we get that $F \in \mathcal{O}_{(X, 0)}$ if and only if for all $a_{i} \neq 0$ it holds $i \in \Gamma_{X}$. If $\alpha \notin \omega_{(X, 0)}$ then there exists $F \in \mathcal{O}_{(X, 0)}$ such that. $\operatorname{res}_{0}(\alpha F)=a_{-n-1} \neq 0$. This implies $-n-1 \in \Gamma_{X}$, which is a contradiction with the hypothesis $n \notin-\Gamma_{X}-1$.

Example 2.7. Let us consider the monomial curve $X$ with parametrization $x_{1}=t^{4}, x_{2}=t^{7}, x_{3}=t^{9}$. Then $e_{0}=4, c=11, \delta=6$. Then $\omega_{X}$ is the $\mathbb{C}$-vector space spanned by $t^{-11}, t^{-7}, t^{-6}, t^{-4}, t^{-3}, t^{-2}, t^{n}, n \geq$ 0 , and the quotient $\omega_{X} / d \mathcal{O}_{(X, 0)}$ admits as $\mathbb{C}$-vector space base the cosets defined by $t^{-11}, t^{-7}, t^{-6}, t^{-4}, t^{-3}$, $t^{-2}, 1, t, t^{2}, t^{4}, t^{5}, t^{9}$. Notice that $\mu(X)=12=2 \delta(X)$. From Proposition 2.5 we get that $x_{1}^{a} \omega_{X}$ is a canonical ideal of $X, a \geq 101$. On the other hand $t^{15} \omega_{X}=\left(x_{1}, x_{3}\right)$ is a canonical ideal as well.

The next step is to find explicit canonical ideals from the resolution of $\mathcal{O}_{(X, 0)}$ when $X$ is a reduced curve singularity of $\left(\mathbb{C}^{3}, 0\right)$. By the Hilbert-Burch theorem we know that there exists a minimal free resolution of $\mathcal{O}_{(X, 0)}$ as an $\mathcal{O}_{\left(\mathbb{C}^{3}, 0\right)}$-module, [7],

$$
0 \longrightarrow \mathcal{O}_{\left(\mathbb{C}^{3}, 0\right)}^{v-1} \stackrel{M}{\longrightarrow} \mathcal{O}_{\left(\mathbb{C}^{3}, 0\right)}^{v} \longrightarrow \mathcal{O}_{\left(\mathbb{C}^{3}, 0\right)} \longrightarrow \mathcal{O}_{(X, 0)} \longrightarrow 0
$$

where $M$ is a $v \times(v-1)$ matrix with entries belonging to the the maximal ideal $\mathcal{O}_{\left(\mathbb{C}^{3}, 0\right)}$ and $I_{X}$ is minimally generated by the maximal minors of $M$. The canonical module of $\mathcal{O}_{(X, 0)}$ is minimally generated by $v-1$ elements. Following [3], [15], we consider a $(2 v-1) \times(2 v-1)$ block-matrix

$$
M_{A}=\left(\begin{array}{c|c}
A & M \\
\hline-M^{\tau} & 0
\end{array}\right)
$$

where $A=\left(a_{i, j}\right)_{i, j=1, \cdots, v}$ is a $v \times v$ skew-symmetric matrix. Notice that $M_{A}$ is also a skew-symmetric matrix, and by the main result of [5] we have a complex

$$
0 \longrightarrow \mathcal{O}_{\left(\mathbb{C}^{3}, 0\right)} \stackrel{\operatorname{Pf}\left(M_{A}\right)^{\tau}}{\longrightarrow} \mathcal{O}_{\left(\mathbb{C}^{3}, 0\right)}^{2 v-1} \stackrel{M_{A}}{\longrightarrow} \mathcal{O}_{\left(\mathbb{C}^{3}, 0\right)}^{2 v-1} \stackrel{\operatorname{Pf}\left(M_{A}\right)}{\longrightarrow} \mathcal{O}_{\left(\mathbb{C}^{3}, 0\right)} \longrightarrow \mathcal{O}_{A}=\mathcal{O}_{\left(\mathbb{C}^{3}, 0\right)} / I_{A} \longrightarrow 0
$$

where $I_{A}$ is the ideal generated by the $\operatorname{Pfaffians} \operatorname{Pf}\left(M_{A}\right)$ of $M_{A}$. This complex is exact if and only if $I_{Z}$ is a height three ideal, if this is the case $\mathcal{O}_{A}$ is a Gorenstein ring, [5].

Proposition 2.8. (i) Let $A$ be a $v \times v$ skew-symmetric matrix such that $I_{A} / I_{X}$ is an $\mathbf{m}_{X}$-primary ideal of $\mathcal{O}_{(X, 0)}$ and $I_{A} / I_{X} \subset \mathbf{m}_{X}^{\mathrm{pn}\left(\mathcal{O}_{(X, 0)}\right)+1}$. Then $I_{A} / I_{X}$ is a canonical ideal of $(X, 0)$.

(ii) For all $t \geq \operatorname{pn}\left(\mathcal{O}_{(X, 0)}\right)$ there exists a $v \times v$ skew-symmetric matrix $A$ with entries of order at least $t$ such that $I_{A} / I_{X}$ is a canonical ideal of $\mathcal{O}_{(X, 0)}$.

Proof. (i) This is a consequence of [5] and Corollary 2.3.

(ii) The ideal $I_{A}$ is generated by the Pfaffians of $M_{A}$, and these elements take the following form, see $[15]$,

1. $F_{i}=(-1)^{i+\frac{(v-1)(v-2)}{2}} \operatorname{det}\left(M_{i}\right), i=1, \ldots, v$, where $M_{i}$ is the matrix removing the $i$-th row of $M$, 
2. $F_{k}=\sum_{1 \leq i<j \leq v}(-1)^{i+j+k+\frac{(v-1)(v-4)}{2}} a_{i, j} \operatorname{det}\left(M_{i, j, k}\right), v+1 \leq k \leq 2 v-1, M_{i, j, k}$ is the matrix removing the $i, j$ rows of $M$ and the $k$ column of $M$.

Notice that $F_{1}, \cdots, F_{v}$ is a system of generators of $I_{X}$. Hence $I_{A} / I_{X}$ is generated by the cosets of $F_{k}$ in $\mathcal{O}_{(X, 0)}, v+1 \leq k \leq 2 v-1$.

Let $\pi: \mathcal{O}_{\left(\mathbb{C}^{3}, 0\right)} \longrightarrow \mathcal{O}_{(X, 0)}$ be the natural projection. Let $J_{X}$ be the ideal generated by the $2 \times 2$ minors of the $v \times 3$ matrix $J a c_{X}$ whose $i$-th row is the gradient vector $\nabla F_{i}=\left(\partial F_{1} / \partial x_{1}, \partial F_{1} / \partial x_{3}, \partial F_{1} / \partial x_{3}\right)$, $i=1, \ldots, v$. The image $\pi\left(J_{X}\right)$ is the Jacobian ideal of $X$. Since the $(X, 0)$ is an isolated curve singularity we have that $\pi\left(J_{X}\right)$ is $\mathbf{m}_{X}$-primary, so $\pi^{-1} \pi\left(J_{X}\right)=I_{X}+J_{X}$ is $\left(x_{1}, x_{2}, x_{3}\right)$-primary.

On the other hand it is easy to prove that

$$
I_{X}+J_{X} \subset K=\left(F_{i} ; 1 \leq i \leq v ; \operatorname{det}\left(M_{i, j, k}\right) ; 1 \leq i<j \leq v ; v+1 \leq k \leq 2 v-1\right),
$$

so $K$ is an $\left(x_{1}, x_{2}, x_{3}\right)$-primary ideal.

Assume that $\operatorname{det}\left(M_{i, j, k}\right)$ is zero divisor of $\mathcal{O}_{(X, 0)}$ for all $\left.1 \leq i \leq v ; 1 \leq i<j \leq v ; v+1 \leq k \leq 2 v-1\right)$. Let $\mathfrak{p}_{1}, \cdots, \mathfrak{p}_{m}$ be the set of minimal primes of $\mathcal{O}_{(X, 0)}$. Then $\mathfrak{p}_{i} \neq \mathbf{m}_{X}, i=1, \cdots, m$, and $K / I_{X} \subset$ $\mathfrak{p}_{1} \cup \cdots \cup \mathfrak{p}_{m}$. Since $K / I_{X}$ an $\mathbf{m}$-primary ideal of $\mathcal{O}_{(X, 0)}$ there is an integer $w$ such that

$$
\left(x_{1}, x_{2}, x_{3}\right)^{w} \subset \frac{K}{I_{X}} \subset \mathfrak{p}_{1} \cup \cdots \cup \mathfrak{p}_{m} .
$$

Hence all element of $\left(x_{1}, x_{2}, x_{3}\right)^{w}$ is a zero divisor of $\mathcal{O}_{(X, 0)}$, but this is not possible because $\mathcal{O}_{(X, 0)}$ is a Cohen-Macaulay ring. We have proved that there exist integers $1 \leq i \leq v ; 1 \leq i<j \leq v ; v+1 \leq k \leq$ $2 v-1)$ such that $\operatorname{det}\left(M_{i, j, k}\right)$ is a non-zero divisor in $\mathcal{O}_{(X, 0)}$.

Let $x \in \mathbf{m} \backslash \mathbf{m}^{2}$ be a non-zerodivisor of $\mathcal{O}_{(X, 0)}$. Let us consider the skew-symmetric matrix such that

$$
\left\{\begin{array}{l}
a_{i, j}=x^{\operatorname{pn}\left(\mathcal{O}_{(X, 0)}\right)}, \\
a_{\alpha, \beta}=0
\end{array} \quad \alpha<\beta,(\alpha, \beta) \neq(i, j) .\right.
$$

Then $F_{k}= \pm x^{\operatorname{pn}\left(\mathcal{O}_{(X, 0)}\right)} \operatorname{det}\left(M_{i, j, k}\right)$ is a non-zero divisor of $\mathcal{O}_{(X, 0)}$. Hence $I_{A} / I_{X}$ is an $\mathbf{m}$-primary ideal of $\mathcal{O}_{(X, 0)}$ contained in $\mathbf{m}^{\mathrm{pn}\left(\mathcal{O}_{(X, 0)}\right)+1}$. By $(i)$ we get that $I_{A} / I_{X}$ is a canonical ideal of $\mathcal{O}_{(X, 0)}$.

Example 2.9. Let us consider a monomial curve singularity $X$ with parametrization $\left(t^{n_{1}}, t^{n_{2}}, t^{n_{3}}\right)$ such that $n_{1}<n_{2}<n_{3}$ and $\operatorname{gcd}\left(n_{1}, n_{2}, n_{3}\right)=1$. Then $e_{0}(X)=n_{1}$ and $\mathcal{O}_{(X, 0)}=\mathbb{C} \llbracket t^{n_{1}}, t^{n_{2}}, t^{n_{3}} \rrbracket \subset \overline{\mathcal{O}_{(X, 0)}}=$ $\mathbb{C} \llbracket t \rrbracket$. The ideal $I_{X}$ is generated by $F_{1}=x_{1}^{r_{3,1}} x_{2}^{r_{3,2}}-x_{3}^{c_{3}}, F_{2}=x_{2}^{r_{1,2}} x_{3}^{r_{1,3}}-x_{1}^{c_{1}}$ and $F_{3}=x_{1}^{r_{2,1}} x_{3}^{r_{2,3}}-x_{2}^{c_{2}}$, where $r_{i, j} \geq 0$ and $c_{i}>0$ is the least integer such that $c_{i} n_{i}=\sum_{i \neq j} r_{i, j} n_{j}, i=1,2,3$, [12]. We assume that $X$ is not a complete intersection, so $r_{i, j}>0$. Then we have that $c_{1}=r_{2,1}+r_{3,1}, c_{2}=r_{1,2}+r_{3,2}$, $c_{3}=r_{1,3}+r_{2,3}$, and $F_{1}, F_{2}, F_{3}$ are the maximal minors of a matrix

$$
M=\left(\begin{array}{cc}
x_{1}^{r_{2,1}} & x_{2}^{r_{1,2}} \\
x_{2}^{r_{3,2}} & x_{3}^{r_{2,3}} \\
x_{3}^{r_{1,3}} & x_{1}^{r_{3,1}}
\end{array}\right)
$$

[18]. Then the matrix $M_{A}$ takes the from

$$
M_{A}=C=\left(\begin{array}{c|c}
A & M \\
\hline-M^{\tau} & 0
\end{array}\right)=\left(\begin{array}{ccc|cc}
0 & a_{1,2} & -a_{1,3} & x_{1}^{r_{2,1}} & x_{2}^{r_{1,2}} \\
-a_{1,2} & 0 & a_{2,3} & x_{2}^{r_{3,2}} & x_{3}^{r_{2,3}} \\
a_{1,3} & -a_{3,2} & 0 & x_{3}^{r_{1,3}} & x_{1}^{r_{3,1}} \\
\hline-x_{1}^{r_{2,1}} & -x_{2}^{r_{3,2}} & -x_{3}^{r_{1,3}} & 0 & 0 \\
-x_{2}^{r_{1,2}} & -x_{3}^{r_{2,3}} & -x_{1}^{r_{3,1}} & & 0
\end{array}\right)
$$


with $a_{i, j} \in \mathbf{m}^{\mathrm{pn}\left(\mathcal{O}_{(X, 0)}\right)}$. Then $I_{A} / I_{X}$ is a canonical ideal generated by the cosets in $\mathcal{O}_{(X, 0)}$ of $a_{2,3} m_{1, i}+$ $a_{1,3} m_{2, i}+a_{1,2} m_{3, i}, i=1,2$, if one of this two elements is a non-zerodivisor of $\mathcal{O}_{(X, 0)}$, see Proposition 2.8 (ii). For instance, we can take $a_{2,3}=x_{1}^{\operatorname{pn}(X)}$ and $a_{i, j}=0$ for $(i, j) \neq(2,3)$, so $\left(x_{1}^{r_{2,1}+\operatorname{pn}(X)}, x_{1}^{\operatorname{pn}(X)} x_{2}^{r_{1,2}}\right)$ is a canonical ideal of $X$.

\section{Canonical ideals and the classification of curve singularities}

In this section we translate the classification of curve singularities to the classification of local Artin Gorenstein rings by means of the quotients by canonical ideals.

First we have to define what generic means in our context. We denote by $S_{t}$ the $\mathbb{C}$-vector space of forms of degree $t$ of $\mathbb{C} \llbracket x_{1}, \ldots x_{n} \rrbracket$.

Proposition 3.1. (i) For all $t \geq 2 \mu(X)+1$ there exists a non-empty Zariski open set $U_{t}(X)$ of $S_{t}$ such that:

(i.1) for all $z \in U_{t}(X), \bar{z} \in \mathcal{O}_{(X, 0)}$ is a degree $t$ superficial element and non-zerodivisor,

(i.2) for all $z_{1}, z_{2} \in U_{t}(X)$

$$
\mathrm{HF}_{\mathcal{O}_{(X, 0)} / \overline{z_{1}} \omega_{(X, 0)}}^{1}=\mathrm{HF}_{\mathcal{O}_{(X, 0)} / \overline{z_{2}} \omega_{(X, 0)}}^{1}
$$

(i.3) for every degree $t$ superficial element $y \in \mathcal{O}_{(X, 0)}$ and for every $z \in U_{t}(X)$ it holds that for all $n \geq 0$,

$$
\mathrm{HF}_{\mathcal{O}_{(X, 0)} / \overline{z_{1}} \omega_{(X, 0)}}^{1}(n) \leq \mathrm{HF}_{\mathcal{O}_{(X, 0)} / y \omega_{(X, 0)}}^{1}(n) .
$$

(ii) For all $z \in U_{t}(X)$ and $t \geq 4 \mu(X)+1$, the socle degree and the length of $\mathcal{O}_{(X, 0)} / \bar{z} \omega_{(X, 0)}$ are constant and analytic invariants of $(X, 0)$ and satisfy

$$
\begin{gathered}
s\left(\mathcal{O}_{(X, 0)} / \bar{z} \omega_{(X, 0)}\right) \leq e_{0}(X)(t-2 \mu(X)-1)+2 \delta(X)+e_{1}(X)+2(1-r), \\
\operatorname{Length}\left(\mathcal{O}_{(X, 0)} / \bar{z} \omega_{(X, 0)}\right)=e_{0}(X) t-2 \delta(X) .
\end{gathered}
$$

Proof. (i) From [19, Proposition 3.2] there exists a non-empty Zariski open set $W_{0} \subset S_{t}$ such that $\bar{z} \in \mathcal{O}_{(X, 0)}$ is a degree $t$ superficial element and non-zerodivisor. From Proposition $2.5(i)$ we know that for all $z \in W_{0}$ we have $\operatorname{Length}\left(\mathcal{O}_{(X, 0)} / \bar{z} \omega_{(X, 0)}\right)=\ell$ with $\ell=t e_{0}(X)-2 \delta(X)$, so $\operatorname{HF}_{\mathcal{O}_{(X, 0)} / \bar{z} \omega_{(X, 0)}}^{1}(n)=\ell$ for all $n \geq \ell$. Hence we only have to consider $n \leq \ell$. Let us consider the upper-semicontinous function

$$
\begin{aligned}
\sigma_{n}: \quad S_{t} & \longrightarrow \\
z & \mapsto \mathrm{HF}_{\mathcal{O}_{(X, 0)} / \bar{z} \omega_{(X, 0)}}^{1}(n) .
\end{aligned}
$$

For each $n=1, \cdots, \ell$, let $W_{n}$ be a non-empty Zariski open set $W_{n} \subset W_{0}$ such that $\sigma_{n}(z)=\min \left\{\sigma_{n}\right\}$ for all $z \in W_{n}$. We set $U_{t}(X)=W_{0} \cap \cdots \cap W_{\ell}$. From the definition of $U_{t}(X)$ it is easy to get $(2)$ and (3).

(ii) From (i.2) we deduce that the socle degree and the length of $\mathcal{O}_{(X, 0)} / \bar{z} \omega_{(X, 0)}$ are constants for all $t \geq 2 \mu(X)+1$ and $z \in U_{t}(X)$. The upper bounds come from Proposition 2.5. Let $\varphi:\left(X_{1}, 0\right) \rightarrow\left(X_{2}, 0\right)$ be an analyic isomorphism between two reduced curve singularities. Let $\varphi_{t}: \mathbf{m}_{X_{1}}^{t} / \mathbf{m}_{X_{1}}^{t+1} \rightarrow \mathbf{m}_{X_{2}}^{t} / \mathbf{m}_{X_{2}}^{t+1}$ be the $\mathbb{C}$-vector space isomorphism induced by $\varphi$. Since $\varphi_{t}\left(U_{t}\left(X_{1}\right)\right) \cap U_{t}\left(X_{2}\right) \neq \emptyset$ we get the last part of the claim.

We write $\ell(X)=e_{0}(X)(4 \mu(X)+1)-2 \delta(X)$. Notice that this is the length of the quotients $\mathcal{O}_{(X, 0)} / \bar{z} \omega_{(X, 0)}$ for $\bar{z} \in U_{4 \mu(X)+1}(X)$.

Definition 3.2. We denote by $\sigma(X)$ the least socle degree of the quotients $\mathcal{O}_{(X, 0)} / I$ where $I$ range the set of canonical ideals $I \subset \mathcal{O}_{(X, 0)}$ with $I \subset \mathbf{m}^{2 \mu(X)+1}$ and $\operatorname{Length}\left(\mathcal{O}_{(X, 0)} / I\right)=\ell(X)$. A canonical ideal $I$ is called deep if $I \subset \mathbf{m}^{2 \mu(X)+1}$, Length $\left(\mathcal{O}_{(X, 0)} / I\right)=\ell(X)$ and $s\left(\mathcal{O}_{(X, 0)} / I\right)=\sigma(X)$. Notice that from Proposition 3.1 deep canonical ideals exist and that $\sigma(X)$ is an analytic invariant. 
Remark 3.3. If $(X, 0)$ is non-singular then we can take $t=1$ in the last identity of Proposition 3.1; in fact we have $s\left(\mathcal{O}_{(X, 0)} / x \mathcal{O}_{(X, 0)}\right)=s(\mathbf{k})=0$. If $(X, 0)$ is Gorenstein then we can take as canonical ideal the whole ring $I=\mathcal{O}_{(X, 0)}$. Then we have $x \in \mathcal{O}_{(X, 0)}$ degree one superficial element, that

$$
s\left(\frac{\mathcal{O}_{(X, 0)}}{x^{t} \mathcal{O}_{(X, 0)}}\right)=s\left(\frac{\mathcal{O}_{(X, 0)}}{x^{e_{0}(X)-1} \mathcal{O}_{(X, 0)}}\right)+t-e_{0}(X)+1,
$$

and Length $\left(\mathcal{O}_{(X, 0)} / x^{t} \mathcal{O}_{(X, 0)}\right)=e_{0}(X) t$, for all $t \geq e_{0}(X)-1$.

Given a non-negative integer $t$ we denote by $\mathbf{H i l b}_{\left(\mathbb{C}^{n}, 0\right)}^{t}$ the Hilbert scheme of length $t$ subschemes $Z$ of $\left(\mathbb{C}^{n}, 0\right)$. We denote by $[Z]$ is the closed point of $\mathbf{H i l b}_{\left(\mathbb{C}^{n}, 0\right)}^{t}$ defined by $Z$. From the universal property of $\mathbf{H i l b}_{\left(\mathbb{C}^{n}, 0\right)}^{t}$ we deduce that any analytic isomorphism $\phi:\left(\mathbb{C}^{n}, 0\right) \longrightarrow\left(\mathbb{C}^{n}, 0\right)$ induces a $\mathbb{C}$-scheme isomorphism $\tilde{\phi}: \mathbf{H i l b}_{\left(\mathbb{C}^{n}, 0\right)}^{t} \longrightarrow \mathbf{H i l b}_{\left(\mathbb{C}^{n}, 0\right)}^{t}$ such that $\tilde{\phi}([Z])=[\phi(Z)]$. Given a canonical ideal $I$ of a reduced curve singularity $(X, 0)$ we denote by $(X, 0)_{I}$ the zero-dimensional scheme $\operatorname{Spec}\left(\mathcal{O}_{(X, 0)} / I\right)$. We know that $(X, 0)_{I}$ is an Artin Gorenstein scheme, [4, Proposition 3.3.18]. It is well-known that two canonical ideals are isomorphic, [4, Theorem 3.3.4]. In the one-dimensional case one can prove more: if $I_{1}, I_{2}$ are canonical ideal there exist non-zero divisors $y_{1}, y_{2} \in \mathcal{O}_{(X, 0)}$ such that $y_{1} I_{1}=y_{2} I_{2}$, [16, Theorem 15.8]. Notice that from the proof of this result we get that for all $z \in U_{t}(X)$ there exists integer $\alpha$ and a regular element $y$ such that $z^{\alpha} I=y z \omega_{(X, 0)}$. The ideals $I_{1}, I_{2}$ are isomorphic as $\mathcal{O}_{(X, 0)}{ }^{-}$ modules, but, in general, there are no analytic isomorphic. Since $K_{t}=x^{t} \omega_{(X, 0)}$ is a canonical ideal for all $t \geq 1, x$ a degree-one superficial element, the Hilbert function of $\mathcal{O}_{(X, 0)} / K_{t}$ varies with $t$.

Proposition 3.4. There exists a non-empty subscheme $\operatorname{Can}(X, 0)$ of the Hilbert scheme $\mathbf{H i l b}_{\left(\mathbb{C}^{n}, 0\right)}^{\ell(X)}$ whose closed points correspond to zero-dimensional Gorenstein schemes $(X, 0)_{I} \subset\left(\mathbb{C}^{n}, 0\right)$ of length $\ell(X)$ and such that $I \subset \mathbf{m}_{X}^{2 \mu(X)+1}$ is a deep canonical ideal.

Proof. Notice that by Proposition 2.5 there exist canonical ideals $I \subset \mathbf{m}_{X}^{2 \mu(X)+1}$ of colength $\ell(X)$. Hence the set of closed points of $\mathbf{H i l b}_{\left(\mathbb{C}^{n}, 0\right)}^{\ell(X)}$ corresponding to such a canonical ideal is non-empty. On the other hand, by standard arguments on the semi-continuity of the dimension of $\mathbf{k}$-vector spaces, there exists a sub-scheme of $\mathbf{H i l b}_{\left(\mathbb{C}^{n}, 0\right)}^{\ell(X)}$ such that its closed points correspond to the quotients $\mathcal{O}_{(X, 0)} / I$ with $I \subset \mathbf{m}_{X}^{2 \mu(X)+1}$. Since $\mathcal{O}_{(X, 0)}$ is a one-dimensional Cohen-Macaulay ring, $I$ is a faithful maximal Cohen-Macaulay $\mathcal{O}_{(X, 0)}$-module. Hence from [4, Proposition 3.3.13] we get that $I$ is a canonical ideal iff $I$ is a type one Cohen-Macaulay $\mathcal{O}_{(X, 0)}$-module. Since the Cohen-Macaulay type is a positive upper semi-continuous function, we get the claim.

Theorem 3.5. Given reduced singularities $\left(X_{i}, 0\right), i=1,2$, the following conditions are equivalent:

(i) there exists an analytic isomorphism $\phi:\left(\mathbb{C}^{n}, 0\right) \longrightarrow\left(\mathbb{C}^{n}, 0\right)$ such that $\phi\left(X_{1}, 0\right)=\left(X_{2}, 0\right)$,

(ii) there exists an analytic isomorphism $\phi:\left(\mathbb{C}^{n}, 0\right) \longrightarrow\left(\mathbb{C}^{n}, 0\right)$ inducing a $\mathbb{C}$-scheme isomorphism $\tilde{\phi}: \operatorname{Can}\left(X_{1}, 0\right) \longrightarrow \operatorname{Can}\left(X_{2}, 0\right)$,

(iii) there exists an analytic isomorphism $\phi:\left(\mathbb{C}^{n}, 0\right) \longrightarrow\left(\mathbb{C}^{n}, 0\right)$ and $\left[\left(X_{1}, 0\right)_{I}\right] \in \operatorname{Can}\left(X_{1}, 0\right)$ such that $\left[\phi\left(\left(X_{1}, 0\right)_{I}\right)\right] \in \operatorname{Can}\left(X_{2}, 0\right)$.

Proof. Given an integer $s \geq 1$ we denote by $(X, 0)_{s}$ the Artin scheme defined by $\mathcal{O}_{(X, 0)} / \mathbf{m}_{X}^{s}$. The implications $(i) \Rightarrow($ ii $) \Rightarrow($ iii $)$ are trivial. Assume that there exist $\left[\left(X_{1}, 0\right)_{I}\right] \in \operatorname{Can}\left(X_{1}, 0\right)$ such that $\left[\phi\left(\left(X_{1}, 0\right)_{I}\right)\right] \in \operatorname{Can}\left(X_{2}, 0\right)$. If $\mu\left(X_{1}\right) \leq \mu\left(X_{2}\right)$ then, since $I \subset \mathbf{m}_{X}^{2 \mu(X)+1}$ we get that $\left(\phi\left(X_{1}, 0\right)\right)_{2 \mu\left(X_{1}\right)+1}=$ $\left(X_{2}, 0\right)_{2 \mu\left(X_{1}\right)+1}$. From the main result of $[8$, Theorem 6] we get that there exists an analytic isomorphism $\varphi:\left(\mathbb{C}^{n}, 0\right) \longrightarrow\left(\mathbb{C}^{n}, 0\right)$ such that $\varphi \phi\left(X_{1}, 0\right)=\left(X_{2}, 0\right)$. If $\mu\left(X_{2}\right)<\mu\left(X_{1}\right)$ then we consider $\left(\phi^{-1}\left(X_{2}, 0\right)\right)_{2 \mu\left(X_{2}\right)+1}=\left(X_{1}, 0\right)_{2 \mu\left(X_{2}\right)+1}$. Again, from the main result of [8, Theorem 6] we get $(i)$. 
An instance of Matlis duality is Macaulay's inverse system: given a Gorenstein Artin algebra $B$ of socle degree $s$, the Macaulay inverse system of $B$ is a polynomial of degree $s$ that encodes several algebraic properties of $B$, see [14]. In the next result we find canonical ideals $I \subset \mathcal{O}_{(X, 0)}$, with suitable socle degree, as the first step to consider the inverse system of a curve singularity.

Proposition 3.6. For all $z \in U_{4 \mu(X)+1}(X), x \in U_{1}(X)$ and $a \geq 0$ it holds

$$
s\left(\frac{\mathcal{O}_{(X, 0)}}{x^{a+1} \bar{z} \omega_{(X, 0)}}\right)=1+s\left(\frac{\mathcal{O}_{(X, 0)}}{x^{a} \bar{z} \omega_{(X, 0)}}\right) .
$$

In particular for suitable integer a we have

$$
s\left(\frac{\mathcal{O}_{(X, 0)}}{x^{a} \bar{z} \omega_{(X, 0)}}\right)=\delta(X)\left(4 e_{0}(X)+3\right)
$$

Proof. The proof of the first identity is standard. The second one follows from Proposition 2.5 (ii) and Proposition 1.1.

Notice that from the last result we can attach to a curve singularity $(X, 0)$ an Artin Gorenstein local ring $B_{X}=\mathcal{O}_{(X, 0)} / x^{a} z \omega_{(X, 0)}$ with socle degree $\delta(X)\left(4 e_{0}(X)+3\right)$. Consequently we can attach to a curve singularity the Macaulay's inverse system $\xi_{X}$ of $B_{X}$ that is a degree $\delta(X)\left(4 e_{0}(X)+3\right)$ polynomial. Hence the algebraic and geometric structure of $X$ is encoded in the polynomial $\xi_{X}$. The development of this fact will be considered elsewhere.

Example 3.7. Let us consider the monomial ring $R=\mathbf{k} \llbracket t^{4}, t^{7}, t^{9} \rrbracket$ of Example 2.7. In this case the polynomial $\xi_{X}$ has degree 144. The explicit computation of this polynomial seems to be very hard, but we can consider a more friendly canonical ideal. Notice that $R=\mathbf{k} \llbracket x_{1}, x_{2}, x_{3} \rrbracket / I$ with $I=\left(x_{1}^{4}-x_{2} x_{3}, x_{2}^{3}-x_{1}^{3} x_{3}, x_{3}^{2}-x_{1} x_{2}^{2}\right)$, and $J=t^{15} \omega_{X}=\left(x_{1}, x_{3}\right)$ is a canonical ideal. Since $x_{1}^{24}\left(x_{1}, x_{3}\right) \subset$ $\mathbf{m}^{2 \mu(X)+1}$, the analytic type of $X$ is determined by the analytic type of the Artin Gorenstein algebra $B=R / x_{1}^{24}\left(x_{1}, x_{2}\right)$, [8]. The ring $B$ has Hilbert function $\left\{1,3,4^{(23)}, 2,1\right\}$, so that $B$ is of multiplicity 99 and socle degree 26 , the inverse system is the degree 26 polynomial:

$$
\begin{aligned}
& 31087081215590400 x_{1} x_{2} x_{3}^{11}+42744736671436800 x_{2}^{8} x_{3}^{6}+284964911142912000 x_{1}^{2} x_{2}^{3} x_{3}^{9}+ \\
& 14248245557145600 x_{1} x_{2}^{10} x_{3}^{4}+341957893371494400 x_{1}^{3} x_{2}^{5} x_{3}^{7}+2849649111429120 x_{1}^{5} x_{3}^{10}+ \\
& 647647525324800 x_{1}^{2} x_{2}^{12} x_{3}^{2}+85489473342873600 x_{1}^{4} x_{2}^{7} x_{3}^{5}+21372368335718400 x_{1}^{6} x_{2}^{2} x_{3}^{8}+ \\
& 2372335257600 x_{1}^{3} x_{2}^{14}+4749415185715200 x_{1}^{5} x_{2}^{9} x_{3}^{3}+14248245557145600 x_{1}^{7} x_{2}^{4} x_{3}^{6}+43176501688320 x_{1}^{6} x_{2}^{11} x_{3}+ \\
& 1781030694643200 x_{1}^{8} x_{2}^{6} x_{3}^{4}+67848788367360 x_{1}^{10} x_{2} x_{3}^{7}+42405492729600 x_{1}^{9} x_{2}^{8} x_{3}^{2}+43176501688320 x_{1}^{11} x_{2}^{3} x_{3}^{5}+ \\
& 94234428288 x_{1}^{10} x_{2}^{10}+3598041807360 x_{1}^{12} x_{2}^{5} x_{3}^{3}+19769460480 x_{1}^{14} x_{3}^{6}+39538920960 x_{1}^{13} x_{2}^{7} x_{3}+ \\
& 19769460480 x_{1}^{15} x_{2}^{2} x_{3}^{4}+1235591280 x_{1}^{16} x_{2}^{4} x_{3}^{2}+4845456 x_{1}^{17} x_{2}^{6}+1700160 x_{1}^{19} x_{2} x_{3}^{3}+85008 x_{1}^{20} x_{2}^{3} x_{3}+24 x_{1}^{23} x_{3}^{2}+ \\
& x_{1}^{24} x_{2}^{2} .
\end{aligned}
$$

\section{References}

[1] A. Altman and S. Kleiman, Introduction to Grothendieck duality theory, Lecture Notes in Mathematics, Vol. 146, Springer-Verlag, Berlin, 1970.

[2] J. Bertin and P. Carbonne, Semi-groupes d'entiers et application aux branches, J. Algebra 49 (1977), no. 1, 81-95.

[3] M. Boij, Gorenstein Artin algebras and points in projective space, Bull. London Math. Soc. 31 (1999), no. 1, 11-16.

[4] W. Bruns and J. Herzog, Cohen-Macaulay rings, revised edition, Cambridge Studies in Advanced Mathematics, vol. 39, Cambridge University Press, 1997. 
[5] D. Buchsbaum and D. Eisenbud, Algebra structures for finite free resolutions, and some structure theorems for ideals of codimension three, Amer. J. Math. 99 (1977), 447-485.

[6] R.O. Buchweitz and G.M. Greuel, The Milnor number and deformations of complex curve singularities, Inv. Math. 58 (1980), 241-281.

[7] L. Burch, On ideals of finite homological dimension in local rings, Math. Proc. Camb. Phil. Soc. 64 (1968), 941-946.

[8] J. Elias, On the analytic equivalence of curves, Math. Proc. Camb. Phil. Soc. 100 (1986), 57-64.

[9] _ Characterization of the Hilbert-Samuel polynomials of curve singularities, Compositio Math. 74 (1990), 135-155.

[10] _ On the deep structure of the blowing-up of curve singularities, Math. Proc. Cambridge Philos. Soc. 131 (2001), 227-240.

[11] S. Goto and K. Watanabe, On graded rings. I, J. Math. Soc. Japan 30 (1978), no. 2, 179-213.

[12] J. Herzog, Generators and relations of abelian semigrous and semigroup-rings, Man. Math. 3 (1970), 153-193.

[13] J. Herzog and E. Kunz, Dir kanonische Modul eines Cohen-Macaulay-Rings, Lecture Notes in Mathematics 238, Springer-Verlag, 1971.

[14] A. Iarrobino, Associated graded algebra of a Gorenstein Artin algebra, Mem. Amer. Math. Soc. 107 (1994), no. 514, viii+115.

[15] A. Iarrobino and V. Kanev, Power sums, Gorenstein algebras, and determinantal loci, Lecture Notes in Mathematics, vol. 1721, Springer-Verlag, Berlin, 1999, Appendix C by Iarrobino and Steven L. Kleiman.

[16] E. Matlis, 1-dimensional Cohen-Macaulay rings, L.N.M. Springer Verlag, 327 (1977).

[17] D.G. Northcott, The reduction number of a one-dimensional local ring, Mathematika 6 (1959), 87-90.

[18] L. Robbiano and G. Valla, On the equations defining tangent cones, Math. Proc. Camb. Phil. Soc. 88 (1980).

[19] J. Sally, Number of generators of ideals in local rings, Lec. Notes in Pure and Appl. Math. New York. 35 (1978).

[20] J.P. Serre, Groupes algébriques et corps de classes, Publications de l'institut de mathématique de l'université de Nancago, VII. Hermann, Paris, 1959.

Juan Elias

Departament d'Àlgebra i Geometria

Universitat de Barcelona

Gran Via 585, 08007 Barcelona, Spain

e-mail: elias@ub.edu 